\title{
Enhancement and inhibition of photoluminescence in hydrogenated amorphous Silicon nitride microcavities
}

\author{
Ali Serpengüzel, Atilla Aydinli, and Alpan Bek \\ Bilkent University, Physics Department, Bilkent, Ankara 06533 Turkey \\ serpen@fen.bilkent.edu.tr
}

\begin{abstract}
A Fabry-Perot microcavity is used for the enhancement and inhibition of photoluminescence in hydrogenated amorphous silicon nitride. The amplitude of the photoluminescence is enhanced 4 times, while its linewidth is reduced 8 times with respect to the bulk hydrogenated amorphous silicon nitride. The transmittance, reflectance, and absorptance spectra of the microcavity were also measured and calculated. The calculated spectra agree well with the experimental ones. (O1997 Optical Society of America

OCIS codes: (230.5750) Resonators; (250.5230) Photoluminescence; (310.0310) Thin films
\end{abstract}

\section{References and links}

1. R. K. Chang and A. J. Campillo, Eds., Optical Processes in Microcavities (World Scientific, Singapore, 1996).

2. E. M. Purcell, " Spontaneous Emission Probabilities at Radio Frequencies, " Phys. Rev. 69, 681 (1946).

3. F. De Martini, G. Innocenti, G. R. Jacobowitz, and P. Mataloni, "Anomalous Spontaneous Emission Time in a Microscopic Optical Cavity," Phys. Rev. Lett. 59, 2955 (1987).

4. H. Yokoyama, S. D. Brorson, "Rate Equation Analysis of Microcavity Lasers," J. Appl. Phys. 66, 4801 (1989).

5. M. S. Ünlü and S. Strite, "Resonant Cavity Enhanced Photonic Devices," J. Appl. Phys. 78, 607 (1995).

6. H. Yokoyama, K. Nishi, T. Anan, H. Yamada, S. D. Brorson, and E. P. Ippen, "Enhanced Spontaneous Emission from GaAs quantum Wells in Monolithic Microcavities," Appl. Phys. Lett. 57, 2814 (1990).

7. S. L. McCall, A. F. J. Levi, R. E. Slusher, S. J. Pearton, and R. A. Logan, "Whispering Gallery Mode Microdisk Lasers," Appl. Phys. Lett. 60, 289 (1992).

8. J. P. Zhang, D. Y. Chu, S. L. Wu, S. T. Ho, W. G. Bi, C. W. Tu, and R. C. Tiberio, "Photonic Wire Laser," Phys. Rev. Lett. 75, 2678 (1995).

9. E. F. Schubert, Y.-H. Wang, A. Y. Cho, 1. W. Tu, and G. J. Zydzik, "Resonant Cavity Light Emitting Diode," Appl. Phys. Lett. 60, 921 (1992).

10. A. M. Agarwal, L. Liao, J. S. Foresi, M. R. Black, X. Duan, and L.C. Kimerling, "Low-loss Polycrystalline Silicon Waveguides for Silicon Photonics," J. Appl. Phys. 80, 6120 (1996).

11. G. Cocorullo, F. G. Della Corte, I. Rendina, C. Minarini, A. Rubino, and E. Terzini, "Amorphous Silicon Waveguides and Light Modulators for Integrated Photonics Realized by Low Temperature Plasma Enhanced Chemical Vapor Deposition," Opt. Lett. 21, 2002 (1996).

12. D. J. Lockwood, "Optical Properties of Porous Silicon," Solid State Commun. 92, 101 (1994).

13. T. Canham, "Silicon Quantum Wire Array Fabrication by Electrochemical and Chemical Dissolution of Wafers," Appl. Phys. Lett. 57, 1046 (1990).

14. F. N. Timofeev, A. Aydinli, R. Ellialtioglu, K. Türkoglu, M. Güre, V. N. Mikhailov, and O. A. Lavrova, "Visible Photoluminescence from SiOx Films Grown by Low Temperature Plasma Enhanced Chemical Vapor Deposition," Solid State Commun. 95, 443 (1995).

15. A. Aydinli, A. Serpengüzel, and D. Vardar, "Visible Photoluminescence from Low Temperature Deposited Hydrogenated Amorphous Silicon Nitride," Solid State Commun. 98, 273 (1996).

16. L. Pavesi, R. Guardini, and C. Mazolleni, "Porous Silicon Resonant Cavity Light Emitting Diodes," Solid State Commun. 97, 1051 (1996).

17. B. T. Sullivan, D. J. Lockwood, H. J. Labbe, and Z.-H. Lu, "Photoluminescence in Amorphous Si/SiOx Superlattices Fabricated by Magnetron Sputtering," Appl. Phys. Lett. 693149 (1996).

18. R. Fisher, in Amorphous Semiconductors, M. H. Brodsky, ed. (Springer-Verlag, Berlin, 1985). pp.159-187.

19. M. H. Brodsky, "Quantum Well Model of the Hydrogenated Amorphous Silicon," Solid State Commun. 36, 55 (1980).

20. G. A. N. Connell, in Amorphous Semiconductors, M. H. Brodsky, ed. (Springer, Berlin, 1985). pp. 73-111. 


\section{Introduction}

Due to their unique optical properties, microcavities continue to attract the attention of the optical spectroscopy community.[1] In a microcavity, two electromagnetic and quantum electrodynamic effects occur. First, the microcavity acts as an optical resonator for light rays with specific wavelengths, which after one round trip, return to their starting position in phase, i.e., resonate in the microcavity. Second, in a microcavity, the photon density of states are enhanced at the cavity resonances, when compared with the continuum of photon states of a bulk sample. The spontaneous emission (luminescence) cross-sections (quantum efficiencies) at the microcavity resonances are larger than the bulk spontaneous emission cross-sections because of the enhanced photon density of states.[2] Also, the luminescence cross-sections in between the microcavity resonances are smaller than the bulk spontaneous emission cross-sections. Alteration (i.e., enhancement and inhibition) of spontaneous emission in planar microcavities has been both observed experimentally [3] and calculated theoretically.[4]

These properties of the microcavities are used in resonant cavity enhanced (RCE) photonic devices, which are wavelength selective and ideal for wavelength division multiplexing (WDM) applications.[5] In vertical cavity surface emitting lasers (VCSEL's),[6] as well as microdisk,[7] and microwire[8] lasers, the lasing threshold is reduced and the mode linewidth is narrowed. Similarly, in RCE light emitting diodes (LED's) the efficiency, brightness, and directivity as well as the cavity finesse and quality factor are enhanced.[9] Also, in RCE photodiodes,[5] the quantum efficiency and the cavity finesse and quality factor are enhanced.

Interest in silicon $(\mathrm{Si})$ as a material for optoelectronics has also increased recently. With modern process techniques, it will be possible to integrate lasers, photodetectors and waveguides on optoelectronic silicon motherboards. Hydrogenated amorphous silicon (a$\mathrm{Si}: \mathrm{H})[10,11]$ has been used for the realization of planar waveguides, which will be able to route and modulate optical signals within such a silicon motherboard. An advantage of a$\mathrm{Si}: \mathrm{H}$ is that, it can be deposited by plasma enhanced chemical vapor deposition (PECVD) on almost any substrate at temperatures below $500 \mathrm{~K}$, which makes it compatible with the microelectronic technology. Another advantage of the a-Si:H[12] as well as porous silicon[13] is that, they also attract interest as a potential optical gain medium because of their room temperature visible electroluminescence and photoluminescence (PL) properties. Recently, we have observed visible PL from a-Si:H, as well as its oxides and nitrides (aSiNx:H) grown by low temperature PECVD.[14,15] Planar microcavity effects on the PL of porous silicon[16] as well as $\mathrm{Si} / \mathrm{SiOx}$ superlattices[17] have already been reported.

In this paper, we report, for the first time, to our knowledge, the enhancement and inhibition of PL in an a-SiNx:H Fabry-Perot microcavity. In our samples, the microcavity was realized by a Au back mirror and an a-SiNx:H-air interface front mirror. First, the thin glass substrates were coated with $\mathrm{Au}$, whose reflectance varied from $50 \%$ for $500 \mathrm{~nm}$ to $98 \%$ for $900 \mathrm{~nm}$. Second, a thin layer of a-SiNx:H was deposited on the Au coated substrates by PECVD at $373 \mathrm{~K}$ with a gaseous mixture of $98 \% \mathrm{~N} 2$ and $2 \% \mathrm{SiH}_{4}$. The flow rate of the gaseous mixture was $180 \mathrm{sccm}$, the radio frequency (RF) power $10 \mathrm{~W}$, and the deposition chamber pressure 1 Torr. The metric thickness $(\mathrm{L})$ of the a-SiNx:H layer was measured with a Veeco DEKTAK 3030 ST surface texture analysis system to be $1400 \pm 50 \mathrm{~nm}$.

\section{Experimental procedures}

The room temperature reflectance and transmittance measurements were made at $0^{\circ}$ with respect to the surface normal using a Varian Cary 5 spectrophotometer with a resolution of 2 $\mathrm{nm}$. 
The room temperature PL spectra were measured with a $1 \mathrm{~m}$ Jobin-Yvon U1000 double spectrometer with a resolution of $0.1 \mathrm{~nm}$, and whose exit slit was equipped with a GaAs photomultiplier tube (PMT) and photon counting electronics. The PL spectra were later corrected for the responsivity of the spectrometer and the PMT. $\mathrm{An}^{+} \mathrm{Ar}^{+}$laser with a wavelength of $514.5 \mathrm{~nm}$ and a power of $420 \mathrm{~mW}$ was focused with a $15 \mathrm{~cm}$ focal length cylindrical lens on the samples. The PL spectra were taken at $0 \pm 3.6^{\circ}$ with the laser at $30^{\circ}$ with respect to the surface normal. During the PL measurements the temperature of the sample is not controlled and there might be local heating due to the poor thermal conductivity of the glass substrate. Local heating reduces the PL efficiency and broadens the PL linewidth. [18] However, the local heating would not considerably affect the general shape and features of the gain spectrum. As will be seen in the PL spectra, even though there might be local heating, we are observing strong PL from the samples and the PL is enhanced by the Fabry-Perot resonances.

While the exact mechanism of the occurrence of the PL in bulk a-SiNx:H is still under discussion, we have suggested in Ref. 15 the use of a quantum confinement model.[19] There, it was proposed that, our samples consist of small a-Si clusters in a matrix of a-SiNx:H. The regions with Si-H and Si-N, having larger energy gaps due to strong $\mathrm{Si}-\mathrm{H}$ and $\mathrm{Si}-\mathrm{N}$ bonds, isolate these a-Si clusters, and form barrier regions around them. The PL originates from these a-Si clusters.

\section{Results}

Figure 1 shows the experimentally measured and theoretically calculated transmittance, and reflectance spectra of the a-SiNx:H microcavity without the Au back mirror. The absorptance spectrum is obtained by subtracting both the reflectance and transmittance spectra from unity, i.e., $100 \%$.

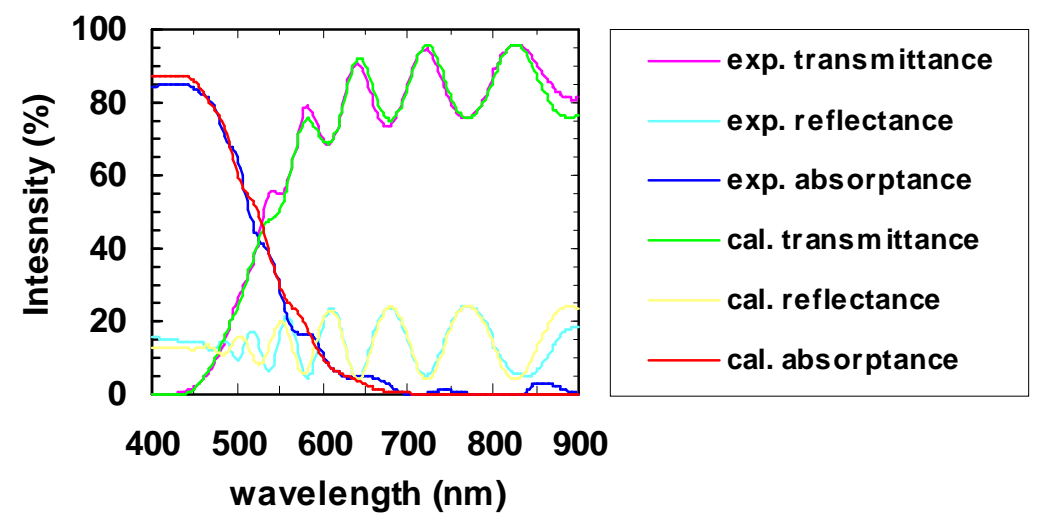

Fig. 1. Experimentally measured and theoretically calculated transmittance, reflectance, and absorptance spectra of the a-SiNx:H microcavity without the Au back mirror.

In the calculations, the total transmitted and the reflected electric fields for each wavelength were obtained using an absorbing Fabry-Perot microcavity model. In this model, we assumed the sample to be a one dimensional absorbing dielectric slab on a glass or on a $\mathrm{Au}$ substrate. The experimentally measured absorptance spectrum was obtained by subtracting both the experimentally measured transmittance and the reflectance spectra from $100 \%$. This experimentally measured absorption spectrum was then incorporated to the calculations of the transmission and reflection electric fields. The calculated transmittance and reflectance intensities at each wavelength were obtained from their respective electric 
fields. The theoretical transmittance and reflectance intensity was subtracted from $100 \%$ to obtain the theoretical absorptance intensity as a final check.

The theoretical fit to the experimental spectra is extremely good. The metric thickness of the a-SiNx:H microcavity is found to be $\mathrm{L}=1376 \mathrm{~nm}$ (which agrees well with the experimental thickness of $1400 \pm 50 \mathrm{~nm}$ ) and the refractive index $n=2.1$. Both the transmittance and the reflectance spectra show Fabry-Perot resonances at wavelengths satisfying the resonance condition $\left(\lambda_{\mathrm{m}}=2 \mathrm{Ln} / \mathrm{m}\right.$, where $\mathrm{m}$ is the quantized mode number). The mode number of these resonances were found to range from $\mathrm{m}=13\left(\lambda_{13}=470 \mathrm{~nm}\right)$ to $\mathrm{m}$ $=7\left(\lambda_{7}=835 \mathrm{~nm}\right)$. The Fabry-Perot resonances have experimental and theoretical linewidths of $\Delta \lambda=35 \mathrm{~nm}$ and quality factors of $\mathrm{Q}=20$ in both the reflectance and the transmittance spectra. Below $600 \mathrm{~nm}$, the resonances start to wash out by the absorption of the a-SiNx:H. The loading of the resonances by the a-SiNx:H absorption stops above $600 \mathrm{~nm}$.

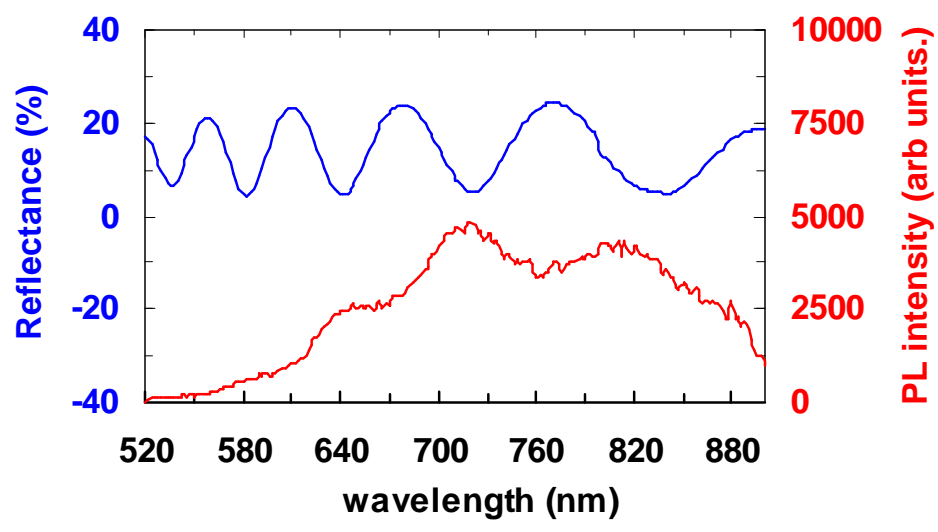

Fig. 2. Reflectance and PL of the a-SiNx:H microcavity without the Au back mirror.

Figure 2 shows the reflectance and the PL of the a-SiNx:H microcavity without the Au back mirror. The PL intensity is modulated by the weak Fabry-Perot resonances, which correlate well with the minima of the reflectance spectrum.

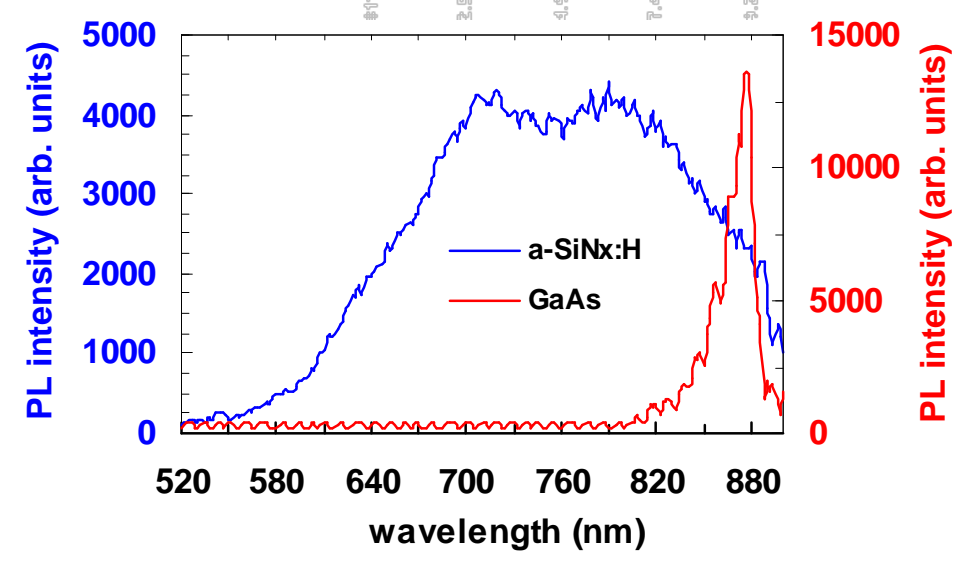

Fig. 3. PL of the a-SiNx:H compared with the PL of GaAs.

\#2274 - \$10.00 US

(C) 1997 OSA
Received August 7, 1997; Revised August 13, 1997 1 September 1997 / Vol. 1, No. 5 / OPTICS EXPRESS 111 
The PL has a broad linewidth $(\mathrm{FWHM}=240 \mathrm{~nm})$ and an external peak efficiency of 3\%, both of which correlate well with the values measured for a-Si:H.[20] Figure 3 shows the PL of the a-SiNx:H and PL of GaAs obtained under the same experimental conditions. These PL spectra have been corrected with respect to the number of absorbed excitation photons. While the external PL peak efficiency of a-SiNx:H is lower than that of GaAs $(10 \%)$, the linewidth of a-SiNx:H (FWHM $=240 \mathrm{~nm})$ is 12 times greater than the linewidth of GaAs (FWHM $=20 \mathrm{~nm}$ ). The high external PL efficiency and the broad gain linewidth of the a-SiNx:H show that, a-SiNx:H has potential as a novel photonic gain medium.

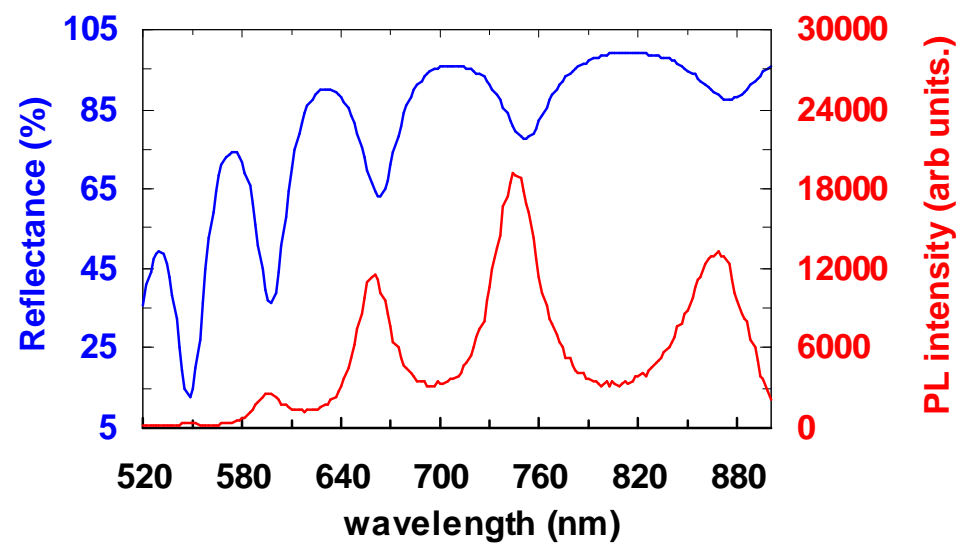

Fig.4. Reflectance and PL of the a-SiNx:H microcavity with the Au back mirror.

Figure 4 depicts the reflectance and the PL of the a-SiNx:H microcavity with the $\mathrm{Au}$ back mirror. The reflectance spectrum of the microcavity with the Au back mirror was also calculated (not shown), using the experimentally observed absorptance spectrum of the a$\mathrm{SiNx}: \mathrm{H}$ and the reflectance spectrum of the $\mathrm{Au}$ mirror, and agrees well with the experimentally observed reflectance spectrum of Fig. 4. These Fabry-Perot resonances have experimental and theoretical linewidths of $\Delta \lambda=25 \mathrm{~nm}$ and quality factors of $\mathrm{Q}=30$ in the reflectance spectra. The metric thickness of the a-SiNx:H microcavity with the Au mirror was found to be $\mathrm{L}=1438 \mathrm{~nm}$ (which agrees well with the experimental thickness of $1400 \pm$ $50 \mathrm{~nm}$ ) and the refractive index $\mathrm{n}=2.1$. The PL is modulated by the strong Fabry-Perot resonances, which correlate well with the reflectance minima. The mode number of these resonances were found to range from $\mathrm{m}=12\left(\lambda_{12}=509 \mathrm{~nm}\right)$ to $\mathrm{m}=7\left(\lambda_{7}=874 \mathrm{~nm}\right)$.

\section{Discussion}

In order to show the enhancement and inhibition of the PL by the Fabry-Perot resonances, we have plotted the PL of the a-SiNx:H microcavity with (X1) and without (X2) the Au mirror in Fig. 5.

The two PL spectra of Fig. 5 were obtained under the same experimental conditions. The PL spectrum of the microcavity without the Au mirror was multiplied by a factor of 2, in order to compare it with the PL spectrum of the microcavity with the Au mirror. When comparing the spectra in Fig. 5, the PL of the microcavity with (X1) the Au mirror has 3 noteworthy features with respect to the PL of the microcavity without (X2) the Au mirror: (1) there is a $2 \mathrm{X}$ increase of the overall spectrum average (i.e., averaging out the Fabry-Perot resonances), (2) there is a $4 \mathrm{X}$ enhancement of the PL peaks, and (3) the PL dips have similar amplitude. These 3 features are noticed with respect to the unmultiplied PL of the microcavity without the Au mirror. 


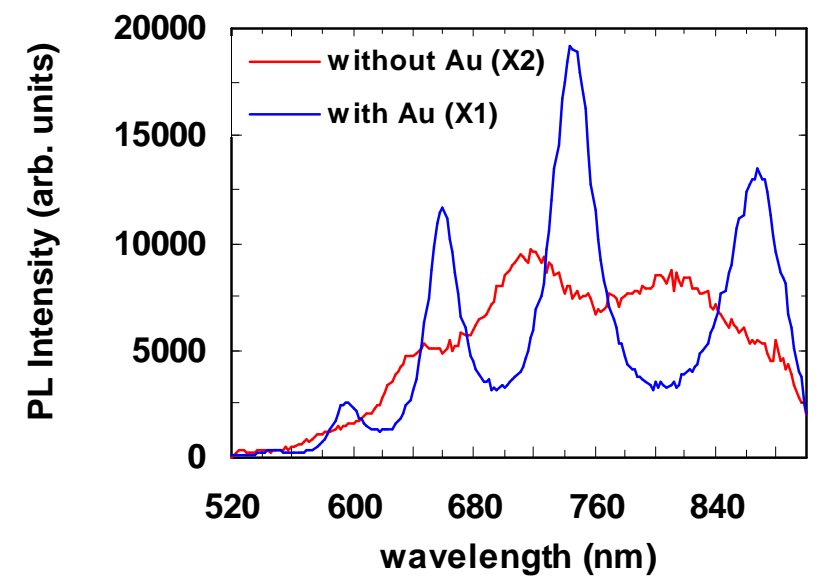

Fig. 5. PL of the a-SiNx:H microcavity with (X1) and without (X2) the Au back mirror.

The $2 \mathrm{X}$ increase is due to the "round trip" of the excitation $\mathrm{Ar}^{+}$laser in the Fabry Perot cavity due to the back Au mirror. Since the wavelength of the $\operatorname{Ar}^{+}$laser $(514.5 \mathrm{~nm})$ is not on a resonance, the input laser light does not resonate in the cavity, which would have enhanced the PL further. The $4 \mathrm{X}$ enhancement at the resonances, are clearly due to the combined effect of the enhancement of the PL by the cavity resonances with that of the input laser reflecting from the back Au mirror. The PL dips having the same amplitude in both spectra is due to the inhibition of the PL in between the resonances.

\section{Conclusions}

In conclusion, a Fabry-Perot microcavity is used for the enhancement and inhibition of PL in a-SiNx:H. The enhancement and inhibition of the PL is understood by the modified photon density of states of the microcavity. The PL is enhanced 4 times at the microcavity resonances, and the linewidth of the PL is narrowed 8 times with respect to the linewidth of the bulk a-SiNx:H, if a Au back mirror is used.

The Fabry-Perot enhancement and inhibition of luminescence in a-SiNx:H opens up a variety of possibilities for optoelectronic and microphotonic applications. A possibility is the use of the a-SiNx:H microcavity as a tunable light source by adjusting the microcavity thickness. Still another possibility is the use of the a-SiNx:H microcavity in RCE photonic devices such as LED's and microlasers.

\section{Acknowledgments}

We would like to thank Murat Güre of Bilkent University, Physics Department for his help with the growth of the samples. We would also like to thank Prof. Richard K. Chang of Yale University, Applied Physics Department for many helpful discussions and reading of the manuscript. We would like to acknowledge the partial support of this research by the Scientific and Technical Research Council of Turkey (TÜBITAK) Grant No: TBAG-1368 and the International Center for Theoretical Physics (ICTP) Grant No: 95-500 RG/PHYS/AS. 